\title{
The Innovation of Information Technology in the Development of Chinese Sports Science
}

\author{
ZHANG Fan ${ }^{1,2 *}$ \\ ${ }^{1}$ Department of Police Skills and Tactics, Nanjing Forest Police College \\ ${ }^{2}$ Sports Science postdoctoral programme, Nanjing Normal University \\ Nanjing 210023, China \\ zhangfan@nfpc.edu.cn
}

\begin{abstract}
Information, as a science, is regarded as one of the four major sciences (life science, material science, information science and Environmental Science) in the world. Combining with the characteristics of information technology, this paper considers the current situation of sports science development, and discusses the development model of sports on the road of being used. From 3 aspects (perceptual to rational, irregular to rule, shallow to deep), this paper discusses the optimization of sports by information technology. The eventual aim is to address competitive sports, guide mass sports, practice school physical education and expand the sports industry by creating unique means of information technology in sports science.
\end{abstract}

Keywords-information technology; sports science; intelligent sports; digital sports; Sports essence; informationization application

\section{INTRODUCTION}

In the new information age, the emphasis of sports research and practice should be satisfied with the development pattern of keeping pace with the times, so that sports can develop towards a "digital sports". Information technology can show complex sports rules in a concise form, and provide strong support for building sports digital models. In an era dominated by information technology, whoever takes the lead in mastering new methods and theories can stand out from the competition. Similarly, in the field of sports, who is the first to grasp the sports culture resources development means, master the scientific training methods and latest technology to improve the level of competitive sports, the development of the new training facilities and sports competitions, the most advanced equipment, who can be in an invincible position in the world in the field of sports.

\section{INFORMATION TECHNOLOGY IS THE DRIVING FORCE OF WORLD DEVELOPMENT}

Information technology refers to the acquisition, delivery, processing and other technologies of information. It is based on microelectronics technology, including communication technology, automation technology, microelectronics technology, photoelectron technology, light guiding technology, computer technology and artificial intelligence machine technology, etc.. Among them, the most important and typical are sensing technology, communication technology and computer technology. They correspond to the sense organs, the nervous system, and the thinking organs of the human body.

\section{A. The rapid rise of information technology}

Information technology (computer technology, sensing technology, communication technology) was first accepted and applied in the society by telephone, radio and so on. With the increase of social demand, remote sensing, optical fiber communication, computer simulation and other new products appeared in the nineteenth Century. In the twentieth Century, information technology has made great breakthroughs in satellite communications, chip software, computer management and ancillary services. The information technology in the twenty-first Century aims at serving the information technology in the twentieth Century, and further improves the development of science and technology, and gradually develops toward digitalization, multifunction, integration and intelligentization. In the future society, information technology will be developed faster, wider and deeper.

\section{B. Information technology has become the dominant factor in} social and economic development

The development of modern information technology is an important symbol of social informatization, and also a dynamic and creative science and technology category in the process of profound changes in the world's economic and social life. For example, in 1998, the contribution rate of information technology to the world economic growth was $14.7 \%$, and the information technology industry will be the leading and the pillar industry of the national economy. Therefore, various industries take information technology as the basis for the rapid development of own field. The birth of science and technology will change the structure of economic industry and make the social economy develop vertically in the direction of informatization [1]. With the development of information technology in the society with high innovation, strong drive and high multiplier, information technology will be the main driving force for social development.

\section{Information technology has high permeability}

Now all fields are speeding up the construction of information technology, making information technology become the "engine" of the whole social economy. First of all, the development of information technology has injected new vitality into other industries. For example, in the sports industry, the application of information technology plays an important role in the scientific training of sports, the enhancement of people's physique, and the promotion of the level of 
competitive sports [2]. Secondly, in combination with other information technology industry constitutes the so-called composite technology, such as sports information technology can make the "essential characteristics of sports, physical fitness, recreation and exercise volition, edify sentiment" is more obvious. Finally, information technology plays an important role in the research and development of new technology. The application of information technology in sports will make sports development in a scientific way.

\section{APPLICATION OF INFORMATION TECHNOLOGY IN SPORTS} FIELD

The information technology has changed the human life style; similarly, the sports informationization also naturally becomes the inevitable request which changes the sports culture. The application of information technology in sports is undergoing a great change from simple applications to deep dependence.

\section{A. Application of information technology in school physical education}

The use of information technology, teachers can put the emphasis and difficulty of movement form, technology project made courseware for students to watch and analyze, interpret by students' questions, create inquiry learning atmosphere. First of all, this approach allows students to learn from a passive attitude to active [3]. Secondly, this application can promote the innovative thinking of students. For example, several basketball shooting simulation scene, let the students observe and analyze and draw the shooting time, body posture, lift arm, wrist, dial refers to the technical details. This combination of information technology teaching model for students can create an ideal sport learning environment and a new way of learning. The use of modern information technology will enable the school sports culture to be disseminated, and lay a foundation for the development of the school sports culture to a higher level.

\section{B. Application of information technology in competitive sports}

Throughout the development of competitive sports, the timeliness of competition news, the accuracy of results and the standardization of venues are getting higher and higher, which ultimately depend on the development of information technology. For example, in the twenty-sixth Olympic Games women's 100 meters final, high-tech computer show champion Devers's shoulder and torso than the runner up Otillo ahead of $0.5 \mathrm{~cm}$, the fair judgment to decide the outcome can be realized only by the high-tech. Information technology in sports training is also important, such as the training of athletes with continuous motion filmed high speed camera, then the system analysis and data processing by computer, or the application of simulation technology, establish and adapt to the individual characteristics of the athletes from the model, and analysis of the lack of training, and put forward the scientific training method. Information technology can detect the athlete's metabolism, such as the combination of human biological rhythms (physical, mental, emotional) software to adjust the training time, so that we can maximize the achievements of athletes.

\section{Application of information technology in mass sports}

Since twenty-first Century, the living needs of people have been greatly improved. In order to satisfy people's demand for scientific fitness, economic fitness, entertainment and fitness, mass sports must develop new fitness equipment with the aid of information technology [4]. The combination of information technology, mass sports has been a significant development, such as the application of virtual reality technology to create virtual reality information in fitness equipment; such as network virtual reality treadmill, digital tennis, boxing etc. The use of network technology, sports fitness instruction site first appeared. Through the guidance of fitness experts, Internet users can sum up a set of effective fitness methods for themselves. This kind of economical and efficient fitness demand brought by information technology makes the sports culture towards the intelligent sports culture rapid growth.

\section{THE OPTIMIZED PATH OF INFORMATION TECHNOLOGY TO SPORTS DEVELOPMENT}

\section{A. From perceptual to rational optimization}

In the dictionary of scientific terms, the explanation of perceptual knowledge and rational knowledge is that perceptual knowledge is the cognition of the phenomena of the people, and has the characteristics of directness and superficiality. Rational knowledge is people's understanding of the nature and laws of things, abstract and indirect, and the real task of cognition is to grasp the nature and laws of things. Therefore, in the sports industry, we must transform perceptual knowledge into rational knowledge.

Since the twentieth Century, the simulation technology and sensor technology have been applied in the field of sports. The purpose is to better analyze the sports structure. But in human movement, there are many factors that are obscure, and even unknown factors exist [5]. As the heart rate telemeter adjusts the athlete's functional response, it is not ideal for the athlete to describe the ECG during the course of movement due to the interference of the electric wave; another example is the size and direction of the jumping force and the angle of joints in the long jump. In the computer plane simulation, we cannot give the correct and comprehensive numerical analysis. The pure mathematical model is limited, and there is no quantitative data for qualitative analysis of the nature and law of sports. Therefore, we can only stay in the perceptual level to understand sports, and have not reached a rational understanding. Therefore, we need to take full advantage of the latest high-tech to develop and develop the essence of sports information technology.

As the development of a simulation considering the full range of diversified data model computer, it can solve the problem of a force signal in different directions and procedures of the system and the external factors that clearly defined, is conducive to a comprehensive and accurate data of movement [6]. For example, data such as jumping, swinging and kicking can be transmitted to computer and computer by sensor technology or communication technology in time, and the actions are calculated, analyzed and corrected.

The development of information technology for sports will make complex movement structures simply expressed. 
Through the "perceptual-quantitative-qualitative-qualitative" analytic hierarchy process of information technology, it can really show the true nature and development law of sports.

\section{B. From irregular to regular transition}

Observing movement from a single point of view is an irregular way of thinking, and analyzing a problem from a systematic and comprehensive perspective is a regular way. In physical education, in order to make students better and faster grasp of sports knowledge, and to train students' thinking ability, we need to systematically observe and think about problems. With the help of information technology, the law of sports can be analyzed from different angles, and the teaching environment can be created purposefully, so that the school sports can realize the change from the irregular to a regular. For example, in a basketball course on "marching lay-up the shot", the teacher displays the action pictures by multimedia technology, so that the students can observe, analyze and ask questions. Students are only interested in the pictures or characters in the picture, but they do not pay attention to the technical essentials. The application of this information technology has no real connection with the problem to be solved, when the environment does not promote learning, and the students' ability to understand physical knowledge cannot be improved. If we use other information technology to teach, the situation is quite different. The teacher first broadcasts the technical action video, then the front, back, left, right, top and bottom of the action are displayed in the screen by 3D animation [7]. Combined with computer software (such as Maya, 3DS, MAX software), the wrist, knee joint, ankle joint and other activities are displayed systematically, then let the students pay attention to the "marching lay-up the shot" wrist, finger, shoulder, jump, turns to the essentials of a series of technical action angle.

In this way, a vivid and comprehensive situation is created. By adjusting the speed of the video, we can observe and analyze the action techniques, so that the students can master the essentials of the technique quickly. In the future, through the skillful use of information technology, this regular development in sports will reflect the value, knowledge and times of sports.

\section{From surface to deep thinking}

The use of information technology (according to the analysis of sports from simple to complex, from outside to inside, principle) on human thinking, perception of sublimation. Since twentieth Century, the field of sports has begun to use the high-speed photography and analysis methods in information technology to track the technical training of athletes and provide scientific training methods for athletes. But we have many of the sports law does not understand, such as running after the kick of the knee why should not jump straight, why cannot use the 45 degree angle of jump [8]. To explore this complex and advanced form of conscious participation, we must use information technology to consider the deep structure of the human body - biomechanics, and to pay attention to the physiological characteristics of the human body. We can regard the human body as a rigid body system with muscle control, and establish the mechanical and mathematical models of human motion. Through the analysis of differential equations of motion, the basic law of human motion is explored. For example, sprint should pay attention to the movement analysis of the center of gravity shift, swing arm, shoulder, horizontal top hip, balances standard. When athletes sprain, they should be deeply analyzed from the physiological structure of the human body. Finding which joints and muscles are involved in exercise, and how scientifically trained these joints and muscles can minimize the risk of sprains.

In order to observe the shape of the joints and obtain the amount of muscle movement, it is necessary to reach the conclusion through high technology. For example, the development of pressure sensors can accurately measure the dynamic forces in the project. In order to obtain human joints, muscles, bones and other different force in different movement of the data, we can simulate the development of a human body joint, the musculoskeletal system of the computer, so that it can show the real human motion system structure. Such simulations can provide an effective tool for the design of new movements, motion error correction, motion analysis and diagnosis, and improvements in muscle strength training methods. The construction of this digital model fully reveals the essence and lows of sports.

\section{CONCLUSION}

Information technology has been an outstanding achievement in sports, especially in service, management and other aspects. But at present, the sports technology culture has not been serialized, systematized and intelligently combed. Based on the nature of the sports industry and sports, this paper presents a new sports information technology, and uses information technology to create an ideal learning environment, exercise environment and leisure environment. By creating unique means of information technology in sports, and developing them (i.e., Information technology applied to sports development). The eventual aim of the era of intelligent sports is to serve competitive sports, guide mass sports, practice school physical education and expand the sports industry. Therefore, China's sports workers should have the courage to face the challenges of the world and the rise of information technology, and strive to improve the overall strength of China's sports.

\section{ACKNOWLEDGMENT}

This work was supported in part by the Project of the Fundamental Research Funds for the Central Universities under Grant LGZD201709, in part by the Project of China Postdoctoral Science Foundation under Grant 2017M611849, in part by Jiangsu Qing LAN Project under Grant 2017, and in part by Nanjing Forest police College Teaching Reform Project under Grant ZD17001 \& YB17001.

\section{REFERENCES}

[1] Yue Huiling. Integration of information technology and physical education curriculum. Journal of Nanjing Sport Institute (NATURAL SCIENCE EDITION), vol. 10, pp. 102-103, March 2011. (In Chinese)

[2] Wu Feng, Fang bin. The dimensions and practical thinking about integration of PE curriculum and information technology at University. Anhui sports science and technology, vol. 26, pp. 66-69, June 2005. (In Chinese) 
[3] Ai Kangwei, GUI Hui, Hong, Wang Bowei, et al. Design and implementation of 3D image analysis, control point measurement and coordinate conversion software. Sports science, vol. 31, pp. 39-43, January 2011. (In Chinese)

[4] Du Yunyun. Using information technology to promote the scientific management of physical education in schools. Fujian computer, vol. 27, pp. 183-184, June 2011. (In Chinese)

[5] Wang Qi. On the application of information visualization software Citespace in sports science. Journal of Jilin Institute of Physical Education, vol. 29, pp. 7-9, June 2013. (In Chinese)
[6] LIU Mei-jin, HUANG Dai-dai. Method of Physical Education Informationization requirement Analysis. Journal of Beijing University of Physical Education, vol. 39, pp. 80-84, April 2016. (In Chinese)

[7] TIAN Lie, SHA Jie, TAN Bo. Current Situation of Informationization Construction of China Sport Colleges. Journal of Beijing University of Physical Education, vol. 39, pp. 33-38, August 2016. (In Chinese)

[8] LI Shang-bin, WANG De-cai, LIU Ying-shuang, et al. Physical education teaching platform design based on the Internet of Things. Journal of Physical Education, vol. 22, pp. 90-94, January 2016. (In Chinese) 